\title{
Linear choosability of graphs
}

\author{
L. Esperet, M. Montassier and A. Raspaud
}

LABRI, Université Bordeaux 1, Domaine Universitaire, 351 cours de la Libération, 33405 Talence, France

A proper vertex coloring of a non oriented graph $G=(V, E)$ is linear if the graph induced by the vertices of two color classes is a forest of paths. A graph $G$ is $L$-list colorable if for a given list assignment $L=\{L(v): v \in V\}$, there exists a proper coloring $c$ of $G$ such that $c(v) \in L(v)$ for all $v \in V$. If $G$ is $L$-list colorable for every list assignment with $|L(v)| \geq k$ for all $v \in V$, then $G$ is said $k$-choosable. A graph is said to be lineary $k$-choosable if the coloring obtained is linear. In this paper, we investigate the linear choosability of graphs for some families of graphs: graphs with small maximum degree, with given maximum average degree, planar graphs... Moreover, we prove that determining whether a bipartite subcubic planar graph is lineary 3-colorable is an NP-complete problem.

Keywords: vertex-coloring, list, acyclic, 3-frugal, choosability under constraints.

\section{Introduction}

Let $G$ be a graph. Let $V(G)$ be its set of vertices and $E(G)$ its set of edges. A proper vertex coloring of $G$ is an assignment $c$ of integers (or labels) to the vertices of $G$ such that $c(u) \neq c(v)$ if the vertices $u$ and $v$ are adjacent in $G$. A $k$-coloring is a proper vertex coloring using $k$ colors. A proper vertex coloring of a graph is linear if the graph induced by the vertices of two color classes is a forest of paths. The linear chromatic number, $\Lambda(G)$, of $G$ is the smallest integer $k$ such that $G$ is lineary $k$-colorable. Linear coloring was introduced by Yuster in [Yus98]. Using technics based on the Lovász Local Lemma, he proved:

Theorem 1 [Yus98] Let $G$ be a graph with maximum degree $\Delta$, then $\Lambda(G)=\mathcal{O}\left(\Delta^{\frac{3}{2}}\right)$.

A linear coloring of graph $G$ is equivalent to an acyclic 3-frugal coloring of $G$ : an acyclic coloring is a vertex coloring such that the graph induced by the vertices of two color classes is a forest and an 3 -frugal coloring is a vertex coloring such that the graph induced by the vertices of two color classes is an induced subgraph with maximum degree 2, i.e. the neighbourhood of any vertex contains the same color at most two times. The lower bound $\Lambda(G) \geq\lceil\Delta / 2\rceil+1$ is a consequence of the 3 -frugality. This bound is reached for simple classes of graphs, such as trees. A greedy coloring of a tree using a deep-first search gives a $(\lceil\Delta / 2\rceil+1)$-coloring. The linear chromatic number of the complete bipartite graph is also easy to compute: if $m \geq n, \Lambda\left(K_{m, n}\right)=\lceil m / 2\rceil+n$.

A graph $G$ is $L$-list colorable if for a given list assignment $L=\{L(v): v \in V(G)\}$ there is a coloring $c$ of the vertices such that $c(v) \in L(v)$ and $c(v) \neq c(u)$ if $u$ and $v$ are adjacent in $G$. If $G$ is $L$-list colorable for every list assignment with $|L(v)| \geq k$ for all $v \in V(G)$, then $G$ is said $k$-choosable. In this paper we focus on linear choosability of graphs. This is, for which value $k$, any list assignement $L$, with $|L(v)| \geq k$ for all $v \in V(G)$ allows a linear coloring of $G$. Let $\Lambda^{l}(G)$ be the smallest integer $k$ such that $G$ is lineary $k$-choosable. We prove:

1365-8050 @ 2005 Discrete Mathematics and Theoretical Computer Science (DMTCS), Nancy, France 
Theorem 2 Let $G$ be an outerplanar graph with maximum degree $\Delta$, then $\Lambda^{l}(G) \leq\left\lceil\frac{\Delta}{2}\right\rceil+2$.

Theorem 3 Let $G$ be a graph with maximum degree $\Delta$, then:

1. If $\Delta \leq 3$, then $\Lambda^{l}(G) \leq 5$.

2. If $\Delta \leq 4$, then $\Lambda^{l}(G) \leq 9$.

Let $G$ be a graph, the maximum average degree of $G$, denoted by $\operatorname{Mad}(G)$ is: $\operatorname{Mad}(G)=\max \{2|E(H)| /|V(H)|, H \subseteq G\}$.

Notice that the maximum average degree of a graph can be computed in polynomial time by using the Matroid Partitioning Algorithm due to Edmonds [Edm65, SU97].

Theorem 4 Let $G$ be a graph with maximum degree $\Delta$ :

1. If $\Delta \geq 3$ and $\operatorname{Mad}(G)<\frac{16}{7}$, then $\Lambda^{l}(G)=\left\lceil\frac{\Delta}{2}\right\rceil+1$.

2. If $\operatorname{Mad}(G)<\frac{5}{2}$, then $\Lambda^{l}(G) \leq\left\lceil\frac{\Delta}{2}\right\rceil+2$.

3. If $\operatorname{Mad}(G)<\frac{8}{3}$, then $\Lambda^{l}(G) \leq\left\lceil\frac{\Delta}{2}\right\rceil+3$.

Since every planar graph $G$ with girth $g(G)$ verifies $M a d(G)<\frac{2 g(G)}{g(G)-2}$, for planar graphs we obtain:

Corollary 1 Let $G$ be a planar graph with maximum degree $\Delta$ :

1. If $\Delta \geq 3$ and $g(G) \geq 16$, then $\Lambda^{l}(G)=\left\lceil\frac{\Delta}{2}\right\rceil+1$.

2. If $g(G) \geq 10$, then $\Lambda^{l}(G) \leq\left\lceil\frac{\Delta}{2}\right\rceil+2$.

3. If $g(G) \geq 8$, then $\Lambda^{l}(G) \leq\left\lceil\frac{\Delta}{2}\right\rceil+3$.

Observe that cycles are lineary 3-choosable; hence, we cannot remove the condition on $\Delta$ in Theorem 4.1.

Theorem 5 Let $G$ be a planar graph with maximum degree $\Delta \geq 9$, then $\Lambda^{l}(G) \leq \Delta+26$.

Theorem 6 Deciding whether a bipartite subcubic planar graph is lineary 3-colorable is an NP-complete problem.

\section{Proof sketches}

In the following, a $k$-vertex (resp. $\geq k$-vertex, $\leq k$-vertex) is a vertex of degree $k$ (resp. $\geq k, \leq k$ ).

Sketch of proof of Theorem 2 In [BGH03], Bonichon, Gavoille and Hanusse show that an outerplanar graph can be decomposed into a spanning tree and a set of edges $M$. Each edge of $M$ links a vertex $v$ with the vertex $f(v)$ defined as follows : if $v$ has a brother at its left, then $f(v)$ is $v$ 's rightmost left brother. Else $f(v)=f(u)$, where $u$ is $v$ 's father (see Figure 1.a).

We color the vertices of $G$ greedily using a deep-first search in the spanning tree. At each step of the algorithm, the vertex that we are trying to color has at most $\lceil\Delta / 2\rceil+1$ colors forbidden by its neighbourhood (at distance at most 2). Thus, there is at least one color in the list of the current vertex that is not forbidden, and this greedy algorithm gives a linear coloring of $G$ given lists of size at least $\lceil\Delta / 2\rceil+2$.

During the process, we distinguish two types of vertices : those which do not have any brother at their left (Type 1 vertices, that can create bichromatic cycles), and the others (Type 2 vertices). 


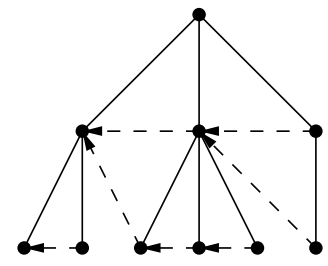

a)

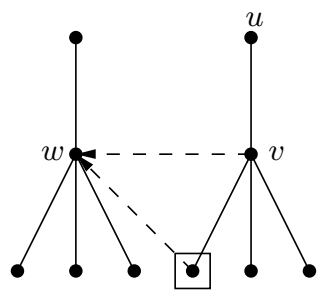

b)

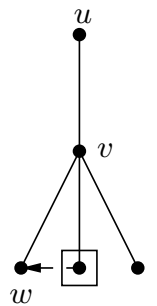

c)

Figure 1: a) An example of the decomposition of an outerplanar graph. b) A vertex of Type 1. $c$ ) A vertex of Type 2.

Sketch of proof of Theorem 3.1 The proof is based on the method of the reducible configurations. Let $G$ be a counterexample with minimum order. We prove that $G$ does not contain the configurations depicted in Figure 2, thus obtaining a contradiction.

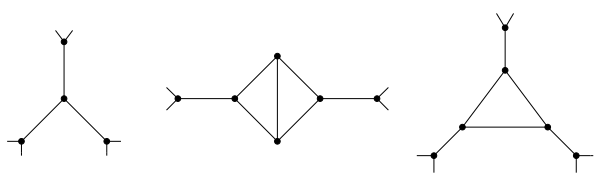

Figure 2: The reducible configurations.

The proof of Theorem 3.2 also uses the method of reducible configurations.

Sketch of proof of Theorem 4.2 Let $H$ be counterexample of minimum order with $\operatorname{Mad}(G)<\frac{5}{2}$. By minimality, $H$ does not contain the following configurations:

1. 1-vertices,

2. two adjacent 2-vertices,

3. a 3-vertex adjacent to three 2-vertices.

We complete the proof of Theorem 4.1 with a discharging procedure. First, we assign to each vertex $v$ a charge $\omega(v)$ equal to its degree. We then apply the following discharging rule:

Rule 1 Each $\geq 3$-vertex gives $\frac{1}{4}$ to each adjacent 2-vertex.

Let $\omega^{*}(v)$ be the new charge of the vertex $v$ after the discharging procedure. Let $v$ be a $k$-vertex (with $k \geq 2$, since $H$ does not contain any 1 -vertices):

- If $k=2$, then $\omega(v)=2$ and $\omega^{*}(v)=2+2 \times \frac{1}{4}=\frac{5}{2}$ since $v$ is not adjacent to any $\leq 2$-vertex and $v$ receives $\frac{1}{4}$ from each adjacent vertex by Rule 1 . 
- If $k=3$, then $\omega(v)=3$ and $\omega^{*}(v) \geq 3-2 \times \frac{1}{4} \geq \frac{5}{2}$ since $v$ is adjacent to at most two 2-vertices and thus gives at most $2 \times \frac{1}{4}$ by Rule 1 .

- If $k \geq 4$, then $\omega(v)=k$ and $\omega^{*}(v) \geq k-k \times \frac{1}{4} \geq \frac{3 k}{4}>\frac{5}{2}$ since $v$ may be adjacent to $k$ 2-vertices.

So, $\forall v \in V(H), \omega^{*}(v) \geq \frac{5}{2}$. Now, observe that $2|E(H)|=\sum_{v \in V(H)} \omega(v)=\sum_{v \in V(H)} \omega^{*}(v)$. Then, by definition of the maximum average degree, we have :

$$
\operatorname{Mad}(H) \geq \frac{2|E(H)|}{|V(H)|}=\frac{\sum_{v \in V(K)} \omega^{*}(v)}{|V(H)|} \geq \frac{5 / 2|V(H)|}{|V(H)|}=\frac{5}{2}
$$

The contradiction with $\operatorname{Mad}(H)$ completes the proof of Theorem 4.2.

The proof of Theorems 4.1 and 4.3 are based on the same method and on similar arguments.

Sketch of proof of Theorem 5 The proof of Theorem 5 is based on van den Heuvel and McGuinness structural lemma [vdHM03] and on the method of reducible configurations.

Sketch of proof of Theorem 6 Let us first show the existence of a special bipartite subcubic planar graph $E(x, y)$ (see Figure 3). The graph $E(x, y)$ is subcubic, planar, and bipartite. Moreover, any linear 3-coloring $c$ of $E(x, y)$ verifies: $c(x)=c(y)$.

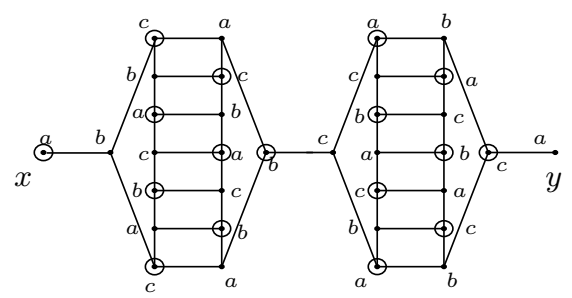

Figure 3: The graph $E(x, y):$ any linear 3-coloring $c$ of $E(x, y)$ verifies: $c(x)=c(y)$

The proof of the NP-completeness proceeds by a reduction to the problem of 3-coloring of planar graphs, that is an NP-complete problem [GJS76]. Given an instance of this problem - a planar graph $G$ -, we need to create a bipartite subcubic planar graph $H$ of a size polynomial in $|V(G)|$ such that $H$ is lineary 3-colorable if and only if $G$ is 3-colorable.

We construct $H$ as follows: For all $k$-vertex $u$ of $G$, we replace $u$ by a binary tree $T_{u}$ with $k$ leaves in $H$ such that each edge $x y$ of $T_{u}$ is replaced by $E(x, y)$. Now, for all adjacent vertex $w$ of $u$ in $G$, we associate one leaf $u_{w}$ in $H$. Finally, for each edge $u v$ of $G$, we add in $H$ an edge linking $u_{v}$ and $v_{u}$ (see Figure 4).

Suppose now that there exists a 3-coloring of $G$. By applying the color of the vertex $v$ to the root of the tree $T_{v}$ in $H$, it is easy to extend the coloring to obtain a linear 3-coloring of $H$ (recall that if the root of $T_{v}$ has the color $a$, then every leaf of $T_{v}$ has the color $a$ and given the color of the root of $T$, it exists a linear 3-coloring of $T$ ). Conversely, if it exists a linear 3-coloring of $H$, it exists a 3-coloring of $G$ by applying the color of the root of the tree $T_{v}$ in $H$ to the vertex $v$ in $G$. 


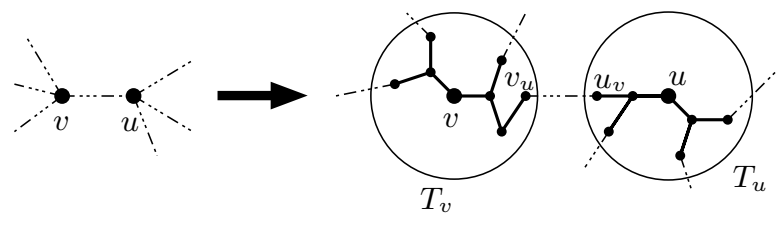

Figure 4: The construction of $H$ from $G$

\section{Conclusion}

We conclude with some open problems:

Problem 1 Is it true that every subcubic graph different from $K_{3,3}$ is lineary 4-colorable?

Problem 2 Minimize the real values $a \leq 1$ and $b$ such that every planar graph with maximum degree $\Delta$ admits a linear $(a \Delta+b)$-coloring?

\section{References}

[BGH03] N. Bonichon, C. Gavoille, and N. Hanusse. Canonical decomposition of outerplanar maps and application to enumeration, coding, and generation. In WG2003: $29^{\text {th }}$ International Workshop, Graph - Theoretic Concepts in Computer Science, pages 81-92, 2003.

[Edm65] J.R. Edmonds. Minimum partition of a matroid into independent subsets. J.Res, Nat. Bur. Standards, 69B:67-72, 1965.

[GJS76] M.R. Garey, D.S. Johnson, and L.J. Stockmeyer. Some simplified NP-complete graph problems. Theor. Comput. Sci., 1(3):237-267, 1976.

[SU97] E.R. Sheiermann and D.H. Ullman. Fractional Graph Theory: A Rational Approach to the Theory of Graphs. Wiley-Interscience series, 1997.

[vdHM03] J. van den Heuvel and S. McGuinness. Coloring the square of a planar graph. Journal of Graph Theory, (42):110-124, 2003.

[Yus98] R. Yuster. Linear coloring of graphs. Discrete Math., (185):293-297, 1998. 
